\title{
Radioimmunoassay and other serologic tests and their application in epidemiological work
}

\author{
M HARBOE \\ University of Oslo, Institute for Experimental Medical Research, Ullevaal \\ Hospital, Oslo 1, Norway
}

\begin{abstract}
Various types of radioimmunoassay (RIA) have been developed for demonstration and quantification of antibodies against Mycobacterium leprae. They may be classified as follows:

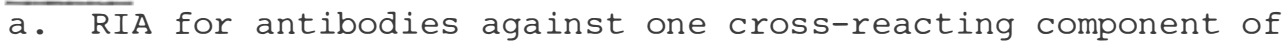
M. leprae.

b. RIA for antibodies against M. leprae specific antigenic determinants.

c. RIA demonstrating antibodies of a particular immunoglobulin class against $\mathrm{M}$. leprae.

The purpose of this paper is to present our current knowledge on these various radioimmunoassays and their informative value in human leprosy and in monitoring the development of systemic mycobacterial infection after experimental inoculation of armadillos. Finally, the plans for further development and tests of these assays in our laboratory will be discussed.
\end{abstract}

RIA for antibodies against one cross-reacting component of $M$. leprae

The RIA for demonstration and quantification of antibodies against M. leprae component 7 (anti-Ml 7) was developed (1, 2) as an example of a test of this kind.

By definition, increased concentrations of anti-Ml 7 are not specific for leprosy but are also expected to occur in other mycobacterial infections.

As with anti-polysaccharide antibodies demonstrated by the passive haemagglutination test of Middlebrook and Dubos (3), antiMl 7 is expected to occur in normal individuals due to stimulation by cross-reacting antigens in environmental mycobacteria.

The amount of anti-Ml 7 antibodies synthesized during mycobacterial infection is expected to be influenced not only by antigenic stimulation due to the infection per se but also by the individual's previous exposure to other mycobacteria containing antigens crossreacting with $M$. leprae antigen 7 .

Strong immune responses may be obtained after primary exposure 
to antigens both in animals and in patients with infectious diseases after previous exposure to cross-reacting antigens (4-8). A test of this kind may therefore be a particularly sensitive indicator of mycobacterial infection. On the other hand, it is essential to establish to what extent the antibody concentration is related to the infectious load in individual patients with a systemic Mycobacterium leprae infection. We have sought to obtain information on this point by studying patients throughout the leprosy spectrum and in experimental models.

Several investigators have found markedly increased concentrations of antibodies against mycobacterial antigens in sera of patients with lepromatous leprosy, whereas such antibodies are detected less frequently and in lower concentrations in patients with tuberculoid leprosy (9-13). It is well established that patients with lepromatous leprosy are characterized by lack of cell mediated immune reactions against $M$. leprae when studied with both in vivo $(10,14)$ and in vitro techniques (15-18). For about a decade, the main view has been that antibody formation and cell mediated immune reactions are inversely related throughout the leprosy spectrum $(19,20)$. Most antibody assays on which this view was based were made by precipitin tests. The RIA for demonstration and quantification of anti-M. leprae 7 antibodies (2) is far more sensitive than precipitin tests. Using this assay, it was found that the median concentration of anti-Ml 7 decreases from the lepromatous to the tuberculoid end of the spectrum, but there is a very striking variation in the antibody concentration of individual sera from patients with similar clinical classification, e.g. in BT leprosy (21).

The reason for this marked variation in antibody content in patients with similar clinical classification is largely unknown, but similar findings were made when antibody assays of markedly increased sensitivity were introduced in studies of other diseases. Specificity for a given disease, and for a particular form of disease, is often lost with increased sensitivity. Precipitating autoantibodies against thyroglobulin were initially demonstrated in Hashimoto's thyroiditis and considered to be specific for this disease. Precipitating anti-thyroglobulin antibodies were, however, only demonstrable in some of the patients. Increased sensitivity was obtained by development of the passive haemagglutination test for anti-thyroglobulin antibodies. Now, the frequency of positive tests in patients with Hashimoto's thyroiditis increased markedly, but at the expense of a distinctly increased frequency of antithyroglobulin antibodies in several other thyroid diseases compared with sera from healthy controls.

The left part of Fig. 1 shows the concentration of anti-M. leprae 7 antibodies in patients throughout the leprosy spectrum; the data are from Yoder et al. (21). In the right part of Fig. 1, the data from the $\mathrm{LL}$ and $\mathrm{BL}$ patients are combined in the left column, and data from BT in the right column. The variation in antibody concentration in individual sera in the two groups is 
indeed striking. If the limit for a positive reaction is set at "30\% of maximal binding by anti-M. leprae", 45 of 53 (84.9\%) of the lepromatous sera were positive whereas 28 of 98 (28.7\%) of the BT sera were positive. These findings are certainly compatible with previous findings in precipitin tests. Increased concentration of anti-Ml 7 antibodies has also been found in $M$. leprae inoculated armadillos (22).

$\%$ of maximal binding

by anti- $\underline{\text { M. }}$ leprae

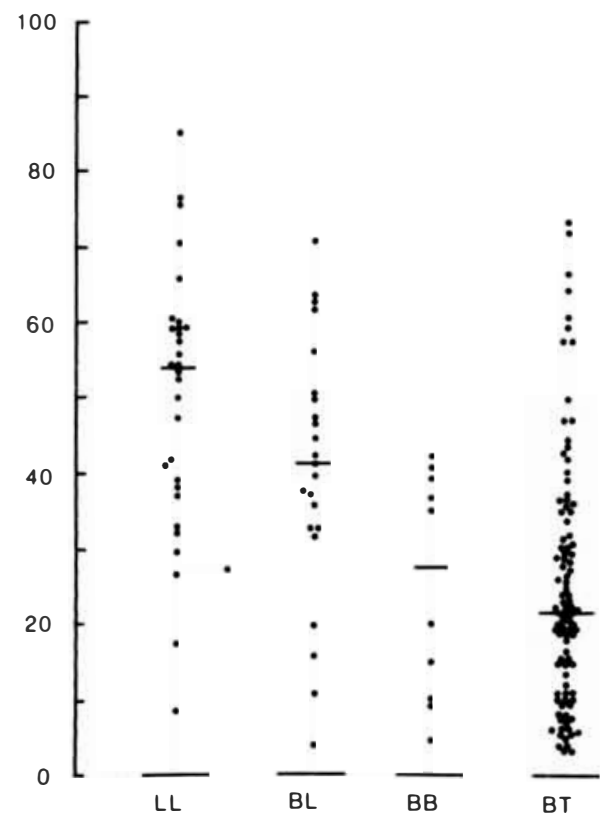

\% of maximal binding

by anti-M. leprae

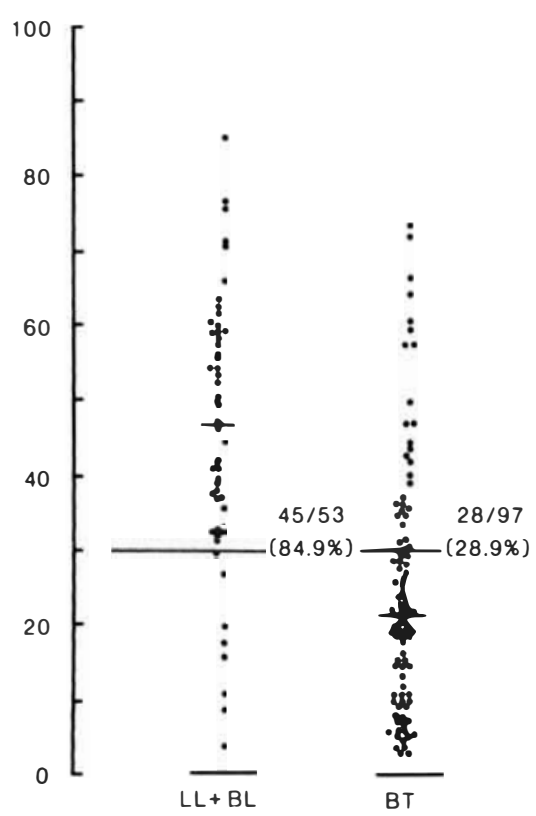

Fig. 1. Antibodies against M. leprae antigen 7 throughout the clinical spectrum of leprosy. In the left part, the data are presented as by yoder et al. (21). In the right part, data from LL and BL patients are pooled to be compared with BT patients. For further explanation, see text.

In view of the marked variation in antibody concentration in patients with similar clinical classification, evidence had to be sought on the following questions:

To what extent is antibody concentration above a certain level a reliable indicator of mycobacterial infection?

How early does this evidence of infection appear in relation to other criteria of mycobacterial infection?

Evidence on these points was sought in cooperation with Dr. C. C. Shepard and Dr. R. J. W. Rees through studies of armadillos inoculated with M. leprae (23). The anti-Ml 7 concentration was assayed 
in serial samples from individual armadillos, and the findings in the antibody assay were correlated with other criteria for development of systemic mycobacterial infection. The animals were regularly examined for nodules at the inoculation site and elsewhere and for acid fast bacilli (AFB) in blood (buffy coat), ear clips, and nasal washings. The data have not been fully analyzed, but are shown in part in Figs. 2 and 3 and Table 1.
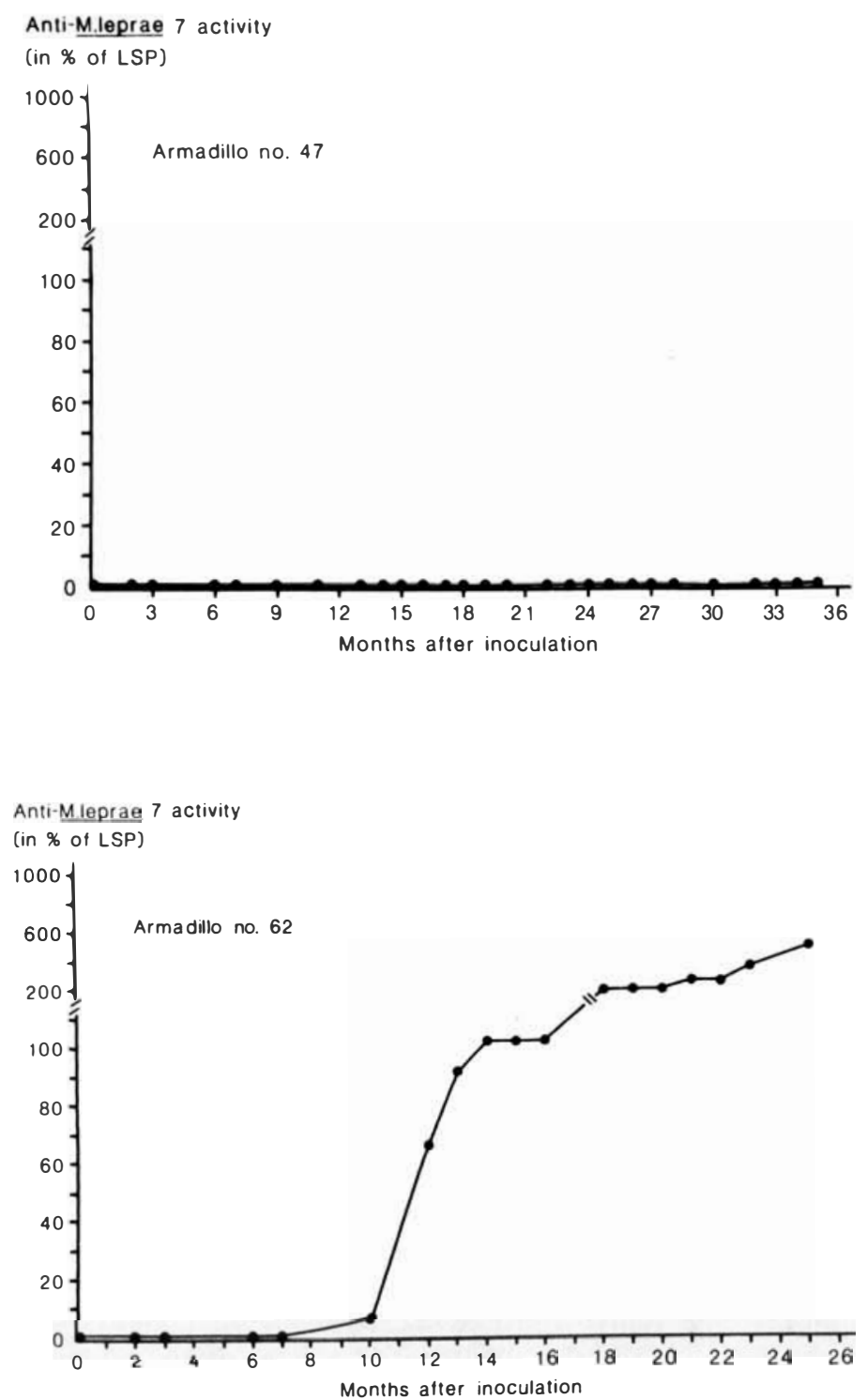

Fig. 2. Assay of antibodies against $M$. leprae antigen 7 in two

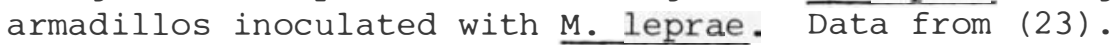


Table 1. Correlation between assay for antibodies against $M$. leprae antigen 7 and development of systemic mycobacterial in $\overline{f e c t i o n}$ in 35 armadillos inoculated with M. leprae. Data from (23).

M. leprae inoculated armadillos

$$
\begin{aligned}
& \text { Evidence of } \\
& \text { Systemic infection } \\
& \begin{array}{rrr|r}
+ & & - \\
\text { Antibody } & 24 & 1 & 25 \\
- & 1 & 9 & 10 \\
\hline 25 & 10 & 35
\end{array}
\end{aligned}
$$

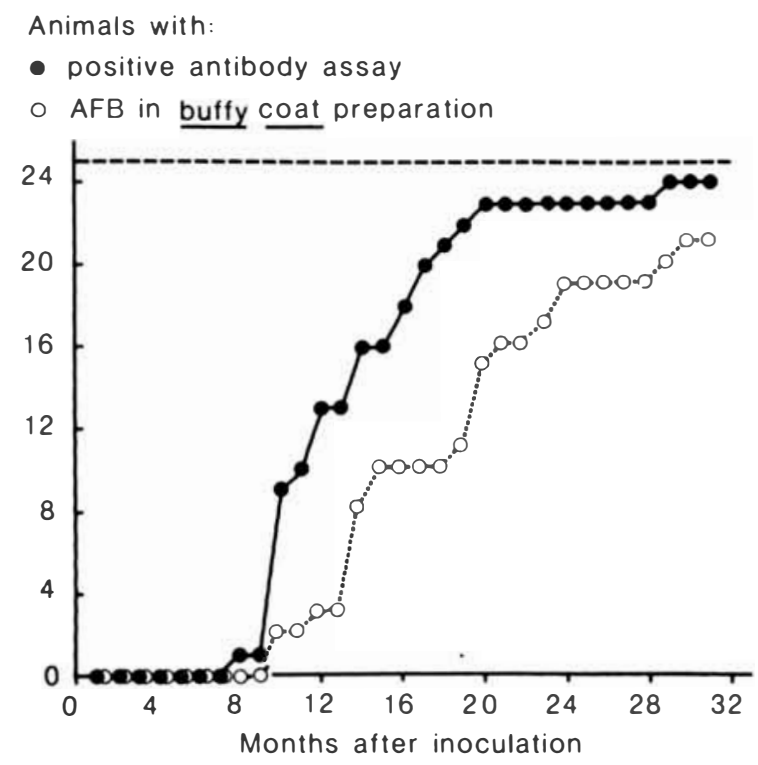

Fig. 3. Actuarial curves showing relationship between occurrence of a positive assay for antibodies against $M$. leprae antigen 7 and demonstration of acid fast bacilli in buffy coat preparations of 25 armadillos with systemic mycobacterial infection after inoculation with M. leprae. Data from (23). 
Figure 2 shows a flat curve from armadillo no. 47 with no signs of antibody formation and the curve of armadillo no. 62 with an initial flat plateau followed by a marked increase in antibody concentration. Table 1 shows that there was a very close correlation between positive findings in the antibody assay and development of systemic mycobacterial infection after the inoculation. In one animal with a false positive result, the assay became positive 24 months after inoculation, i.e. later than in all but one of the animals that developed systemic infection. Fig. 3 shows actuarial curves where occurrence of a positive antibody assay (anti-Ml 7 concentration above $20 \%$ of activity in the control lepromatous serum pool) was compared with demonstration of AFB in buffy coat preparations.

A nodule at the inoculation site was usually the first sign of development of mycobacterial infection in the inoculated animals. This occurred slightly before development of a positive antibody assay. A positive result in the antibody assay was, however, obtained before the appearance of nodules at other sites. A positive antibody assay was also obtained earlier than the other criteria of systemic mycobacterial infection which became positive in the following order: AFB in peripheral blood, AFB in ear clips, and $\mathrm{AFB}$ in nasal washings.

Increasing concentration of anti-M. leprae 7 antibody is thus an early indicator of infection in the armadillo. It should be borne in mind, and it may be an essential point, that the histology of the lesions in the armadillos correspond closely to lepromatous and not to tuberculoid leprosy in man (24-26). Experiments are in progress to assay separately IgM- and IgG anti-M. leprae antibodies in the armadillos to see if the introduction of immunoglobulin class specific assays makes an earlier demonstration of infection by immunological testing possible.

Time course of development of cell mediated- and humoral immunity in different forms of leprosy

In lepromatous forms of the disease, cell mediated immune reactions against compcnents of $M$. leprae are absent or weak (10, 14-18). Although the concentration varies in individual cases, antibodies are usually demonstrable in these patients $(9-13,21)$. In experimental animals and in patients with lepromatous forms of the disease, antibodies will thus be the most sensitive indicator of infection. Additional data are needed on their development in relation to the development of the infection and, thus, on to what extent they are suitable as reliable indicators of subclinical infection developing towards the lepromatous part of the spectrum.

In the tuberculoid part of the spectrum, cell mediated immune reactions and hypersensitivity against components of $\mathrm{M}$. leprae are a characteristic feature. Our data show that antibodies also occur in these forms of the disease $(21,27)$ and may be of considerable 
interest in further analysis and studies of such patients (28). The time relationship between development of cell mediated- and humoral immunity in these individuals needs further study.

Assays for antibodies against M. leprae specific antigenic determinants

Antigenic determinants highly specific for M. leprae may occur on components that also contain cross-reacting determinants. Determinants of the former kind were first demonstrated by Kronvall et al. using crossed immunoelectrophoresis (CIE) with lepromatous serum as antibody reagent in the top gel. Kronvall et al. demonstrated M. leprae specific determinants on one component of M. leprae (29). Similar determinants may occur on several components, but exact information on this point is still lacking. Assays for antibodies reacting with such determinants may generally be developed based on the principle of absorption, by which antibodies reacting with cross-reacting determinants are neutralized. or removed from the system. Assays of this kind have been developed on the basis of various techniques, as a fluorescent antibody absorption test by Abe $(30,31)$ and a RIA in our laboratory (32). Assays of antibodies against lipid antigens on M. leprae (33) should be thoroughly studied in this context.

The findings in our RIA strongly indicate that a significant proportion of antibodies occurring in lepromatous leprosy serum react with determinants highly specific for $M$. leprae. By successive absorptions with sonicates of other mycobacteria such as BCG, M. avium, and M. nonchromogenicum, antibodies reacting with the latter mycobacteria may be removed whereas reactivity with $M$. leprae remains to a great extent (32). The evaluation of the specificity of the test is, however, not yet completed. The need for absorption with sonicates of other mycobacteria may vary in different regions, depending on the frequency of occurrence and infection with other mycobacteria. Information on specificity tests applying the assay on sera from patients with various infectious diseases and other diseases including hypergammaglobulinaemia is still insufficient in our RIA and in published data on the other tests.

A conflict between specificity and sensitivity has to be foreseen. We know that cross-reacting determinants are strongly immunogenic, but more information is needed on the immunogenicity of $M$. leprae specific determinants. It may be that a combination of $\overline{r e l a-}$ tive specificity and taking the absolute antibody concentration into account may be most rewarding, as has been clearly demonstrated by Closs et al. in lymphocyte stimulation tests with M. leprae antigen 7 and tuberculin PPD (18).

Monoclonal antibodies produced by the hybridoma technology are expected to be "the next generation" of anti-M. leprae reagents. By definition, they will be homogeneous and react with individual determinants on immunogenic components of M. leprae. If, or when 
monoclonal antibodies reacting with determinants highly specific for M. leprae become available, techniques will immediately be available for precise identification of acid fast bacilli as $\mathrm{M}$. leprae and for demonstration of $M$. leprae specific antibodies in patient sera by competitive binding assays.

Immunoglobulin class specific assays

Solid phase radioimmunoassays (SRIA) for demonstration of IgG-, IgA-, and IgM antibodies against $M$. leprae have been developed by Melsom et al. (34). The ELISA technique has also been established in our laboratory for demonstration of immunoglobulin class associated anti-M. leprae antibodies (unpublished observations). The specificity of these tests with regard to reactivity of the antibodies with the various components of $M$. leprae has not yet been established. The evidence obtained so far indicates that the assay for IgG antibodies against M. leprae detects both antibodies against M. leprae antigen 7 and other components.

The immunoglobulin class specific assays and the anti-Ml 7 assay gave similar findings when applied to sera from patients throughout the leprosy spectrum. The median antibody concentration was higher in patients with lepromatous than in tuberculoid leprosy, and again there was a wide variation between antibody content in individual patients with similar clinical classification.

One finding was of particular interest in connection with epidemiological studies of leprosy. A group of patients with "strictly indeterminate leprosy" (35) was studied (36). These were patients classified as clinically indeterminate leprosy having a single or a few vague hypopigmented skin lesions, and the histological features were also typical of indeterminate leprosy. In the IgG anti-M. leprae assay, this group was not significantly different from the control group consisting of people exposed to M. leprae through work in a leprosy hospital but without any clinical signs of leprosy. In the IgA anti-M. leprae assay, the median antibody concentration was higher in the indeterminate group than in the control group, but with considerable overlap. In the IgM anti-M. leprae assay, there was an increased antibody concentration in the group of indeterminate leprosy patients and no overlap with the control group.

So far, the group of patients termed "strictly indeterminate leprosy" has been considered to be a group of individuals infected with M. leprae and showing very weak clinical sign of the disease due to lack of stimulation of the immune system at this stage (35). The findings of Melsom et al. (36) indicate that humoral immune reactions have been induced in these patients although they have rather low concentrations of anti-M. leprae antibodies. Production of IgM antibodies against the infectious agent may thus be an important and early diagnostic sign in leprosy where the causative organism is an obligate intracellular parasite characterized by 
extremely slow growth.

Additional investigations are needed in this area. They are critically dependent upon accurate clinical observation and classification combined with histological studies and antibody assays. The second major but difficult point is suitable selection of controls from the same socioeconomic background without clinical signs of leprosy in whom it may be difficult to evaluate the extent of exposure to M. leprae. Additional criteria are needed for diagnosis of indeterminate leprosy to make it more reliable and consistent in different institutions. Many of these lesions are known to heal spontaneously, whereas others develop into tuberculoid or lepromatous leprosy. Reliable demonstration of such a lesion being caused by infection with $\underline{M}$. leprae is required to establish the frequency of the various subsequent courses with sufficient accuracy.

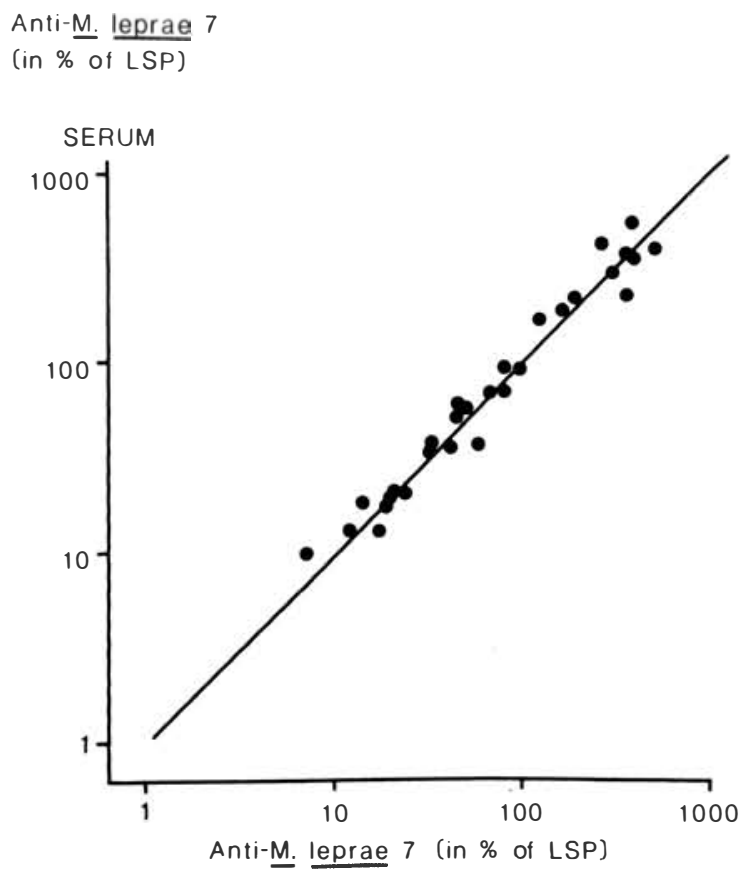

blood

Fig. 4. Comparison between assay for antibodies against $\underline{M}$. leprae antigen 7 in serum and frozen and thawed venous blood from 29 leprosy patients. Data from (37).

\section{Application under field conditions}

Applicability of tests under field condititions depends upon several factors. Simple tests may be carried out in the field or in laboratories close to field areas. If the tests are more complicated, 
transport of materials for study to sufficiently well-equipped laboratories becomes essential. Antibody assays would be greatly simplified if they could be carried out on whole blood frozen immediately after sampling in the field.

Figure 4 shows anti-M. leprae 7 antibody activity determined by radioimmunoassay in serum and frozen and thawed whole blood from a group of 29 leprosy patients (37).. The findings obtained with frozen and thawed whole blood correspond closely to those obtained with serum from the same patients. Menzel et al. found that determination of antibodies against M. leprae antigen 7 by RIA, and IgG antibodies against a total M. leprae sonicate by solid phase RIA and the ELISA technique could all be made on both whole blood samples and serum samples. In addition, it was demonstrated that frozen finger-prick blood could be used for antibody assays as well as venous blood. These assays, at least, should thus be applicable to test material obtained in field work.

Further development of currently available antibody assays

Assays for anti-M. leprae 7 antibodies, antibodies against M. leprae specific determinants, and immunoglobulin class specific anti-M. leprae antibodies represent assays of three principally different kinds.

These assays provide different types of information related to various questions in need of investigation to obtain a better and sufficient knowledge of the epidemiology of leprosy. At present, one should not rely on a single type of assay but pursue several in parallel to establish their respective value and drawbacks.

The specificity of the tests needs further study to define which components of $\mathrm{M}$. leprae are reactive in the various tests. For the fluorescent antibody absorption test $(30,31)$ as well as radioimmunoassay (32) and other tests to be developed for demonstration of antibodies against $M$. leprae specific determinants, it is required to define the extent to which they are really specific for demonstration of antibodies reacting with $\mathrm{M}$. leprae and not with other species of mycobacteria. By definition, M. leprae specific assays should be negative in individuals in non-endemic countries. Additional studies are needed on sera from individuals in nonendemic countries with tuberculosis, infection with "atypical mycobacteria", other infectious diseases, and polyclonal hypergammaglobulinemia. Careful studies of a few sera of this kind giving false positive reactions are expected to be particularly valuable for characterization and improvement of the specificity of the assays.

The relation between specificity and sensitivity needs further study and consideration. When the specificity is increased, going more and more for M. leprae specific determinants, the immunogenicity of these determinants becomes a critical issue, and it may be difficult to steer between Scylla and Charybdis. My prediction 
tends to go in the direction of attempting to develop a relatively specific test combined with an indicator of antibody concentration as in the lymphocyte stimulation test with a purified component of M. leprae developed by Closs et al. (18) .

The use of immunoglobulin class specific assays certainly needs to be further pursued, particularly in indeterminate leprosy in man and in careful long-term studies in experimental models such as $M$. leprae inoculated armadillos and apes.

The relationship between antigenic load during mycobacterial infection and antibody content needs further study, again both in leprosy patients and relevant experimental models. Information related to this point will also be sought by determination of antibody concentration in individuals harbouring "atypical "mycobacteria as saprophytes and pathogens.

Concerning antibodies against cross-reacting components of $\mathrm{M}$. leprae, it is not known if other specificities can provide additional information.' Due to the findings already made, anti-M. leprae 7 will continue to be a main object of our studies using two main principles:

Simultaneous studies of cell mediated- and humoral immunity working with purified and defined components of the bacillus.

Combined studies in a leprosy-endemic and a leprosy non-endemic area which is required to evaluate and have sufficient control of the specificity of the assays.

\section{Acknowledgements}

The original work in our laboratory referred to in this paper was supported by grants from Anders Jahre's Fund for the Promotion of Science, the Norwegian Council for Science and the Humanities, and the Immunology of Leprosy (IMMLEP) component of the UNDP/World Bank/WHO Special Programme for Research and Training in Tropical Diseases. We acknowledge in particular the close cooperation and provision of material from the Armauer Hansen Research Institute (AHRI) and the All-Africa Leprosy and Rehabilitation Centre (ALERT), Addis Ababa, Ethiopia.

\section{References}

1 Harboe M, Closs O, Bjorvatn B, Kronvall G, Axelsen NH. Antibody response in rabbits to immunization with Mycobacterium leprae. Infect Immun, 1977, 18, 792-805.

2 Melsom R, Naafs B, Harboe $M$, Closs $O$. Antibody activity against Mycobacterium leprae antigen 7 during the first year of DDS treatment in lepromatous (BL - LL) leprosy. Lepr Rev, 1978, $49,17-29$.

3 Middlebrook G, Dubos RJ. Specific serum agglutination of erythrocytes sensitized with extracts of tubercle bacilli. 
J Exp Med, 1948, 88, 521-528.

4 Eisen HN, Little JR, Steiner LA, Simms ES. Degeneracy in the secondary immune response: stimulation of antibody formation by cross reacting antigens. Israel J med Sci, 1969, 5, 338351 .

5 Closs O, Kronvall G. Experimental murine leprosy IX. Antibodies against Mycobacterium lepraemurium in $\mathrm{C} 3 \mathrm{H}$ and $\mathrm{C} 57 \mathrm{BL}$ mice with murine leprosy and in patients with lepromatous leprosy. Scand J Immunol, 1975, 4, 735-740.

$6 \mathrm{H} \varnothing \mathrm{iby}$. Cross-reactions between Pseudomonas aeruginosa and thirty-six other bacterial species. Scand J Immunol, 1975, $\underline{4}$, Suppl 2, 187-196.

$7 \mathrm{H} \varnothing$ iby N. Normally occurring precipitating antibodies against Pseudomonas aeruginosa. Prevalence, specificities and titres. Scand J Immunol, 1975, 4, Suppl 2, 197-202.

8 Mshana RN, Closs O, Harboe M. Antibody response in rabbits to Mycobacterium bovis BCG. Scand J Immunol, 1979, 9, 175-182.

9 Almeida JO. Serology in leprosy. Bull Wld Hlth Org, 1970, 42 , 673-702.

10 Turk JL, Bryceson ADM. Immunological phenomena in leprosy and related diseases. Adv Immunol, 1971, 13, 209-266-

11 Myrvang B, Feek CM, Godal T. Antimycobacterial antibodies in sera from patients throughout the clinico-pathological disease spectrum of leprosy. Acta Path Microbiol Scand Sect B, 1974, 82, 701-706.

12 Axelsen NH, Harboe M, Closs O, Godal T. BCG antibody profiles in tuberculoid and lepromatous leprosy. Infect Immun, 1974, 9, 952-958.

13 Kronvall G, Bjune G, Stanford J, Menzel S, Samuel D. Mycobacterial antigens in antibody responses of leprosy patients. Int J Leprosy, 1975, 43, 299-306.

14 Rees RJW. The significance of the lepromin reaction in man. Prog Allergy, 1964, 8, 224-258.

15 Godal T, Myklestad B, Samuel DR, Myrvang B. Characterization of the cellular immune defect in lepromatous leprosy: A specific lack of circulating Mycobacterium leprae-reactive lymphocytes. Clin Exp Immunol, 1971, 9, 821-831.

16 Godal T, Myrvang B, Fröland SS, Shao J, Melaku G. Evidence that the mechanism of immunological tolerance ("central failure") is operative in the lack of host resistance in lepromatous leprosy. Scand J Immunol, 1972, 1, 311-321.

17 Myrvang B, Godal T, Ridley DS, Frölānd SS, Song YK. Immune responsiveness to Mycobacterium leprae and other mycobacterial antigens throughout the clinical and histopathological spectrum of leprosy. Clin Exp Immunol, 1973, 14, 541-553.

18 Closs O, Reitan LJ, Negassi K, Harboe M, Belehu A. In vitro stimulation of lymphocytes in leprosy patients, healthy contacts of leprosy patients, and subjects not exposed to leprosy. Comparison of an antigen fraction prepared from Mycobacterium leprae and tuberculin purified protein derivative. Scand J 
Immunol (In press).

19 Godal T, Myrvang B, Stanford J-L, Samuel DR. Recent advances in the immunology of leprosy with special reference to new approaches in immunoprophylaxis. Bull Inst Pasteur, 1974, 72, 273-310.

20 Bullock WE. Leprosy: A model of immunological perturbation in chronic infection. J Inf Dis, 1978, 137, 341-354.

21 Yoder L, Naafs B, Harboe M, Bjune G. Antibody activity against Mycobacterium leprae antigen 7 in leprosy: Studies of variation in antibody content throughout the spectrum and on the effect of DDS treatment and relapse in BT leprosy. Lepr Rev, $1979,50,113-121$.

22 Harboe M, Closs O, Rees RJW, Walsh GP. Formation of antibody against Mycobacterium leprae antigen 7 in armadillos. J Med Microbiol, 1978, 11, 525-535.

23 Harboe M, Closs O, shepard CC, Pacific J, Rees RJW. Long term studies of anti-M. leprae 7 antibodies in $M$. leprae inoculated armadillos. (In preparation).

24 Kirchheimer WF, Storrs EE. Attempts to establish the armadillo (Dasypus novemcinctus Linn.) as a model for the study of leprosy. I. Report of lepromatoid leprosy in an experimentally infected armadillo. Int J Leprosy, 1971, 39, 693-702.

25 Kirchheimer $\mathrm{WF}$, Storrs $\mathrm{EE}, \mathrm{Binford} \mathrm{CH}$. Attempts to establish the armadillo (Dasypus novemcinctus Linn.) as a model for the study of leprosy. II. Histopathologic and bacteriologic postmortem findings in lepromatoid leprosy in the armadillo. Int J Leprosy, 1972, 40, 229-242.

26 Rees RJW. Personal communication.

27 Harboe M, Closs O, Bjorvatn B, Bjune G. Antibodies against BCG antigen 60 in mycobacterial infection. Brit med J, 1977, 2, 430-433.

28 Harboe M, Closs O, Reitan LJ, Draper P. Demonstration of antibodies reacting with different determinants on Mycobacterium leprae antigen 7. Int J Leprosy (In press).

29 Kronvall G, Stanford JL, Walsh GP. Studies of mycobacterial antigens, with special reference to Mycobacterium leprae. Infect Immun, 1976, 13, 1132-1138.

30 Abe M, Izumi S, Saito T, Mathur SK. Early serodiagnosis of leprosy by indirect immunofluorescence. Lepr India, 1976, 48, 272-276.

31 Abe M, Yoshimo Y, Saikawa K, Saito T. Fluorescent leprosy antibody absorption (FLA - ABS) test for detecting subclinical infection of Mycobacterium leprae. Int J Leprosy, 1980, 48, 109-119.

32 Harboe M, Closs O, Bjune G, Kronvall G, Axelsen NH. Mycobacterium leprae specific antibodies detected by radioimmunoassay. Scand J Immunol, 1978, 7, 111-120.

33 Brennan PJ, Barrow WW. Evidence for species-specific lipid antigens in Mycobacterium leprae. Int J Leprosy, 1980, 48, 382387. 
34 Melsom R, Harboe M, Duncan ME, Bergsvik H. IgA and IgM antibodies towards $M$. leprae in cord sera and in patients with leprosy: An indicator of intrauterine infection in leprosy. Scand J Immunol (in press).

35 Myrvang B, Godal T, Feek CM, Ridley DS, Samuel DR. Immune response to Mycobacterium leprae in indeterminate leprosy patients. Acta Path Microbiol Scand Sect B, 1973, 81, 615-620.

36 Melsom R, Harboe M, Myrvang B, Godal T, Belehu A. Immunoglobulin class specific antibodies to $M$. leprae in leprosy patients, including the indeterminate group and healthy contacts as a step in the development of methods for sero-diagnosis of leprosy. Clin Exp Immunol (Submitted for publication).

37 Menzel S, Harboe M, Belehu A, van der Meulen J. Suitability of whole blood for the demonstration of antibodies to Mycobacterium leprae proven in three different assays. Bull Wld Hlth org. (To be submitted for publication). 